\title{
n-Point Functions for the Rectangular Ising Ferromagnet
}

\author{
D. B. Abraham^
}

Department of Theoretical Chemistry, Oxford University, Oxford OX1 3TG, England

\begin{abstract}
A new representation for the n-point functions of the Planar Ising ferromagnet is given. Below the critical temperature the boundary conditions are toroidal; the state is a superposition of the extremal invariant ones, with equal weights.
\end{abstract}

\section{Introduction}

This paper presents the final results which are needed to write down the $n$-point function of the rectangular Ising ferromagnet in an explicit way. As was explained in the first paper [1], this can be done once all matrix elements of spin operators between any eigenvectors of the transfer matrix have been given. In [1] and [2], matrix elements from the vacua to any excited state were considered. The method for completing the problem is quite obvious, but the fact that a Wick theorem still obtains is not; it is also highly significant for the truncation properties of the $n$-point functions [3]. The results of this series of papers have found application in the rigorous determination of critical indices [4], in heuristic remarks on the equation of state [5] and in the analysis of the density profile between phases [6].

\section{Generalised Matrix Elements}

Let functions associated with the generalised matrix elements be defined by

$$
\begin{gathered}
F\left(\left(e^{i \beta}\right)_{m} \mid\left(e^{i \alpha}\right)_{m+1, n}\right) \\
=M^{n / 2} \exp i\left\{\sum_{1}^{m}\left(\beta_{j}+\theta\left(\beta_{j}\right)\right)+\sum_{m+1}^{n}\left(\alpha_{j}+\theta\left(\alpha_{j}\right)\right)\right\} \\
\cdot\left\langle\Phi_{-}\left|G_{\alpha_{n}} \ldots G_{\alpha_{m+1}} G_{-\beta_{m}}^{+} \ldots G_{-\beta_{1}}^{+}\right| \Phi_{+}\right\rangle .
\end{gathered}
$$

\footnotetext{
* Supported by the Fonds National Suisse de la Recherche Scientifique, by the National Science Foundation Grant No. PHY 76-17191, and by the National Research Council of Canada Grant No. NRC A 9344
} 
By using the linear dependence relationship

$$
G_{-\beta}^{+}=\sum_{\alpha}\langle\beta \mid \alpha\rangle\left(\cos (\theta(\beta)-\theta(\alpha)) G_{-\alpha}^{+}+i \sin (\theta(\beta)-\theta(\alpha)) G_{\alpha}\right)
$$

with

$$
\langle\beta \mid \alpha\rangle=2 / M\left(e^{i(\beta-\alpha)}-1\right)
$$

the following recurrence relationship may be derived:

$$
\begin{aligned}
& F_{M}\left((t)_{m} \mid(z)_{m+1, n}\right) \\
& =\sum_{z_{m}} \frac{1}{M\left(z_{m} / t_{m}-1\right)}\left(1-\frac{\Theta\left(z_{m}\right)}{\Theta\left(t_{m}\right)}\right) F_{M}\left((t)_{m-1} \mid(z)_{m, n}\right) \\
& \quad+\sum_{j=m+1}^{n}(-1)^{j-m-1} \frac{z_{j} t_{m}}{z_{j} t_{m}-1}\left(1+\frac{1}{\Theta\left(z_{m}\right) \Theta\left(t_{j}\right)}\right) F_{M}\left((t)_{m} \mid \Delta_{j}(z)_{m+1, n}\right) .
\end{aligned}
$$

The first summation is over distinct $z_{m}$ such that $z_{m}^{M}=1$. The relevant object in the limit $M \rightarrow \infty$ is given by

$$
\begin{aligned}
& F\left((t)_{m} \mid(z)_{m+1, n}\right)=\frac{\mathscr{P}}{2 \pi i} \int_{C_{1}} \frac{d z_{m}}{z_{m}} \frac{1}{\left(z_{m} / t_{m}-1\right)}\left(1-\frac{\Theta\left(z_{m}\right)}{\Theta\left(t_{m}\right)}\right) \\
& F\left((t)_{m-1} \mid(z)_{m, n}\right) \\
& \quad+\sum_{j=m+1}^{n}(-1)^{j-m+1} \frac{z_{j} t_{m}}{z_{j} t_{m}-1}\left(1+\frac{1}{\Theta\left(t_{m}\right) \Theta\left(z_{j}\right)}\right) F\left((t)_{m-1} \mid \Delta_{j}(z)_{m+1, n}\right) .
\end{aligned}
$$

The solution of (2.5) will be developed separately for $T>T_{C}$ and $T<T_{C}$ using an inductive ansatz.

By analogy with the introduction of the operator $Y_{+}$in the previous two papers, consider the operator $Y_{-}$defined on a dense substance of $L^{2}\left(S_{1}\right)$ by

$$
\left(Y_{-} f\right)(t)=\frac{\mathscr{P}}{\pi i} \int_{C_{1}} \frac{d z}{z} \frac{1}{z / t-1}\left(1-\frac{\Theta(z)}{\Theta(t)}\right) f(z) .
$$

This may be extended to $\bigotimes_{1}^{n} L^{2}\left(S_{1}\right)$ by

$$
\left(Y_{-} f\right)\left((z)_{n}\right)=\frac{\mathscr{P}}{\pi i} \int_{C_{1}} \frac{d t_{1}}{t_{1}} \frac{1}{t / z_{1}-1}\left(1-\frac{\Theta(t)}{\Theta(z)}\right) f\left(t,(z)_{2, n}\right) .
$$

Clearly the norm satisfies $\left\|Y_{-}\right\| \leqq 2$.

Consider first the case $T>T_{C}$ : since $F\left((z)_{2 n}\right)$ is known, when $m=1$ in (2.5) we have

$$
\begin{aligned}
F\left(t \mid(z)_{2,2 n}\right)= & \sum_{2}^{2 n}(-1)^{j} F\left(\Delta_{j}(z)_{2,2 n}\right)\left[\frac{1}{2}\left(Y_{-} f_{-}\right)\left(t, z_{j}\right)\right. \\
& \left.+\frac{z_{j} t}{z_{j} t-1}\left(1+\frac{1}{\Theta\left(z_{j}\right) \Theta(t)}\right)\right],
\end{aligned}
$$


where the pair contraction function $f_{-}$is expressed in terms of the Wiener-Hopf factorisation (see [1], Appendix B) of $\Theta(z)$ by

$$
f_{ \pm}(z, t)=\frac{z t}{z t-1}\left(\Theta_{+}^{-1}(z) \Theta_{-}^{-1}(t) \pm \Theta_{+}^{-1}(t) \Theta_{-}^{-1}(z)\right)
$$

The additional function $f_{+}$will be encountered in the following. Using the properties of the factorisation (see [1], Appendix B) it follows that

$$
\begin{aligned}
& \left(Y_{-} f_{-}\right)\left(t, z_{j}\right)=2 f_{+}\left(t, z_{j}\right)-\frac{2 z_{j} t}{z_{j} t-1}\left(1+\frac{1}{\Theta\left(z_{j}\right) \Theta(t)}\right), \\
& \left(Y_{-} f_{+}\right)\left(t, z_{j}\right)=2 f_{-}\left(t, z_{j}\right) .
\end{aligned}
$$

Insertion of (2.10) into (2.8) gives

$$
F\left(t \mid(z)_{2,2 n}\right)=\sum_{2}^{2 n}(-1)^{j} f_{+}\left(t, z_{j}\right) F\left(\Delta_{j}(z)_{2,2 n}\right) .
$$

This result suggests the inductive ansatz

$$
\begin{aligned}
F\left((t)_{m} \mid(z)_{m+1,2 n}\right)= & \sum_{1}^{m-1}(-1)^{j-m} f_{-}\left(t_{m}, t_{j}\right) F\left(\Delta_{j}(t)_{m-1} \mid(z)_{m+1,2 n}\right) \\
& +\sum_{m+1}^{2 n}(-1)^{j-m+1} f_{+}\left(t_{m}, z_{j}\right) F\left((t)_{m-1} \mid \Delta_{j}(z)_{m+1,2 n}\right) .
\end{aligned}
$$

In order to test whether this satisfies (2.5), for $m \geqq 2,(2.10)$ and (2.11) are needed; then (2.13) is readily verified by induction on $m$, for any $n \geqq 1$.

If $T<T_{C}$ and $m=1$ then the expansion (4.18) of Paper I should be used with contraction function and initial condition as follows:

$$
\begin{aligned}
f_{ \pm}(z, t) & =\frac{z t}{z t-1}\left(\Theta_{+}^{-1}(z) t^{-1} \Theta_{-}^{-1}(t) \pm \Theta_{+}(t) z^{-1} \Theta_{-}^{-1}(z)\right), \\
F(z) & =z \Theta_{+}^{-1}(z) \Theta_{+}(0) m^{*} .
\end{aligned}
$$

Then from (2.5) it follows that

$$
\begin{aligned}
F\left(t \mid(z)_{2,2 n+1}\right)= & -\frac{1}{2}\left(Y_{-} F\right)(t) F\left((z)_{2,2 n+1}\right) \\
& +\sum_{j=2}^{2 n+1}(-1)^{j}\left\{F\left(z_{j}\right) \frac{1}{2}\left(Y_{-} F\right)\left(t, \Delta_{1 j}(z)_{2 n+1}\right)\right. \\
& \left.+\frac{z_{j} t}{z_{j} t-1}\left(1+\frac{1}{\Theta\left(z_{j}\right) \Theta(t)}\right) F\left(\Delta_{j}(z)_{2 n+1}\right)\right\} .
\end{aligned}
$$

The results needed here are that

$$
\begin{aligned}
\left(Y_{-} f_{-}\right)(t, z)= & 2 f_{+}(t, z)-\frac{2 z t}{z t-1}\left(1+\frac{1}{\Theta(z) \Theta(t)}\right) \\
& +2 \Theta_{+}(0) \Theta^{-1}(t) z \Theta_{+}(z)^{-1}, \\
\left(Y_{-} f_{+}\right)(t, z)= & 2 f_{-}(t, z)-2 \Theta_{+}(0) \Theta^{-1}(t) z \Theta_{+}(z)^{-1},
\end{aligned}
$$


and

$$
\left(Y_{-} F\right)(z)=2 F(z) \text {. }
$$

The terms involving $F\left(z_{j}\right)$ on the right side of (2.16) and (2.17) cancel in (2.15) by appealing to the properties of Pfaffians, giving the result

$$
\begin{aligned}
F\left(t \mid(z)_{2,2 n+1}\right)= & \sum_{2}^{2 n+1}(-1)^{j} F\left(z_{j}\right) F\left(t \mid \Delta_{1 j}(z)_{2 n+1}\right) \\
& -F(t) F\left((z)_{2,2 n+1}\right)
\end{aligned}
$$

where $\quad F\left(t \mid \Delta_{1}(z)_{2 n}\right)=\sum_{2}^{2 n}(-1)^{k} f_{+}\left(t, z_{k}\right) F\left(\Delta_{1 k}(z)_{2 n}\right)$

the final Pfaffian being evaluated according to Paper I of the series. An inductive ansatz analogous to (2.13) can now be made, and established for $T<T_{C}$; it is (2.36) of Theorem 2.

The matrix elements

$$
\begin{aligned}
& F_{M}^{x}\left(\left(e^{i \beta}\right)_{m} \mid\left(e^{i \alpha}\right)_{m+1, n}\right)=M^{n / 2} \exp i\left\{\sum_{1}^{m}\left(\beta_{j}+\theta\left(\beta_{j}\right)\right)\right. \\
& \left.\quad+\sum_{m+1}^{n}\left(\alpha_{j}+\theta\left(\alpha_{j}\right)\right)\right\}\left\langle\Phi_{-}\left|G_{\alpha_{n}} \ldots G_{\alpha_{m+1}} \sigma_{1}^{x} G_{-\beta_{m}}^{+} \ldots G_{-\beta_{1}}^{+}\right| \Phi_{+}\right\rangle
\end{aligned}
$$

are calculated in the appropriate limit as $M \rightarrow \infty$ by precisely the same procedure as in Paper II.

For $T>T_{C}$ we have the equation

$$
\begin{aligned}
& F^{x}\left((t)_{m} \mid(z)_{m+1,2 n+1}\right) \\
& =\sum_{j=1}^{m}(-1)^{m-j-1} \Theta\left(z_{j}\right)^{-1} F\left(\Delta_{j}(t)_{m} \mid(z)_{m+1,2 n+1}\right) \\
& \quad+\frac{\mathscr{P}}{2 \pi i} \int_{C_{1}} \frac{d t}{t} \Theta(t) F\left((t)_{m}, t \mid(z)_{m+1,2 n+1}\right) \\
& =\sum_{j=1}^{m}(-1)^{m+j-1}\left\{\Theta\left(t_{j}\right)^{-1}+\frac{\mathscr{P}}{2 \pi i} \int_{C_{1}} \frac{d t}{t} \Theta(t) f_{-}\left(t, t_{j}\right)\right\} \\
& \quad \cdot F\left(\Delta_{j}(t)_{m} \mid(z)_{m+1,2 n+1}\right) \\
& \quad+\sum_{j=m+1}^{n}(-1)^{m+j} \frac{\mathscr{P}}{2 \pi i} \int_{C_{1}} \frac{d t}{t} \Theta(t) f_{+}\left(t, z_{j}\right) \\
& \quad \cdot F\left((t)_{m} \mid \Delta_{j}(z)_{m+1,2 n+1}\right) .
\end{aligned}
$$

But we have the results

$$
\begin{aligned}
& \frac{\mathscr{P}}{2 \pi i} \int_{C_{1}} \frac{d t}{t} \Theta(t) f_{+}(t, z)=\Theta_{-}(\infty) \Theta^{-1}(z), \\
& \frac{\mathscr{P}}{2 \pi i} \int_{C_{1}} \frac{d t}{t} \Theta(t) f_{-}(t, z)=\Theta_{-}(\infty) \Theta_{-}^{-1}(z)-\Theta^{-1}(z),
\end{aligned}
$$


from which the results given in the Theorem 1 below follow. The analogous results for $T<T_{C}$ are obtained by conducting the expansion of the Pfaffian in line 1 of (2.23) according to (2.36). Using the results

$$
\frac{\mathscr{P}}{2 \pi i} \int_{C_{1}} \frac{d t}{t} \Theta(t) f_{+}(t, z)=-z \Theta_{+}^{-1}(z) \Theta_{+}(0)
$$

and

$$
\frac{\mathscr{P}}{2 \pi i} \int_{C_{1}} \frac{d t}{t} \Theta(t) f_{-}(t, z)=-\frac{1}{\Theta(z)}+z \Theta_{+}^{-1}(z) \Theta_{+}(0)
$$

together with the normalisation

$$
\frac{1}{2 \pi i} \oint_{C_{1}} \frac{d t}{t} \Theta(t) F(t)=m^{*}
$$

then gives the appropriate part of Theorem 2. The results are as follows:

Theorem 1. If $\mathscr{I}(\Theta)=0\left(T>T_{C}\right)$ then for $0 \leqq m \leqq 2 n+1$

$$
F\left((z)_{m} \mid(z)_{m+1,2 n+1}\right)=0
$$

whereas

$$
F\left((z)_{m} \mid(z)_{m+1,2 n}\right)=\sum_{1}^{2 n}(-1)^{j} f\left(z_{1}, z_{j}\right) F\left(\Delta_{1 j}(z)_{m} \mid(z)_{m+1,2 n}\right)
$$

with

$$
f\left(z_{i}, z_{j}\right)=f_{+}\left(z_{i}, z_{j}\right)\left[\operatorname{resp} . f_{-}\left(z_{i}, z_{j}\right)\right]
$$

for $1 \leqq i \leqq m, m+1 \leqq j \leqq 2 n$ (resp. $1 \leqq i \leqq m$ and $1 \leqq j \leqq m$ or $m+1 \leqq i \leqq 2 n, m+1 \leqq j$ $\leqq 2 n)$.

Here

$$
f_{ \pm}(z, t)=\frac{z t}{z t-1}\left(\Theta_{+}^{-1}(z) \Theta_{-}^{-1}(t) \pm \Theta_{+}^{-1}(t) \Theta_{-}^{-1}(z)\right) .
$$

on the other hand

$$
F^{x}\left((z)_{m} \mid(z)_{m+1,2 n}\right)=0
$$

whereas

$$
\begin{aligned}
F^{x}\left((z)_{m} \mid z_{m+1,2 n+1}\right)= & \sum_{j=1}^{2 n+1} \Theta_{-}(\infty)(-1)^{j} \Theta_{-}^{-1}\left(z_{j}\right) \\
& \cdot F\left(\Delta_{j}(z)_{m} \mid(z)_{m+1,2 n+1}\right),
\end{aligned}
$$

where the generalised Pfaffian is given by (2.30) and (2.31).

Theorem 2. If $\mathscr{I}(\Theta)=-1\left(T<T_{C}\right)$ then for $0 \leqq m \leqq 2 n$

$$
F\left((z)_{m} \mid(z)_{m+1,2 n}\right)=0
$$


whereas

$$
F\left((z)_{m} \mid(z)_{m+1,2 n+1}\right)=\sum_{j=1}^{2 n+1}(-1)^{j} F\left(z_{j}\right) F\left(\Delta_{j}(z)_{m} \mid(z)_{m+1,2 n+1}\right)
$$

the second matrix element factor on the right hand side being given by (2.22) and (2.23) of the previous theorem, with

$$
\begin{aligned}
& f_{ \pm}(z, t)=\frac{z t}{z t-1} \Theta_{+}^{-1}(z) t^{-1} \Theta_{-}^{-1}(t) \pm \Theta_{+}^{-1}(t) z^{-1} \Theta_{-}^{-1}(z), \\
& F(z)=\Theta_{+}(0) m^{*} z \Theta_{+}^{-1}(z) .
\end{aligned}
$$

On the other hand

$$
F^{x}\left((z)_{m} \mid(z)_{m+1,2 n+1}\right)=0
$$

whereas

$$
F^{x}\left((z)_{m} \mid(z)_{m+1,2 n}\right)=F\left((z)_{m} \mid(z)_{m+1,2 n}\right)
$$

the right hand side being given by (2.22) and (2.23), with the pair contraction function $f_{ \pm}$given by

$$
f_{ \pm}(z, t)=\frac{z t}{z t-1}\left(\Theta_{+}(z)^{-1} t^{-1} \Theta_{-}(t)^{-1} \pm \Theta_{+}(t)^{-1} z^{-1} \Theta_{-}(z)^{-1}\right) .
$$

Remarks. 1. The matrix elements are written in terms of Pfaffians which are generalised further to include symmetric contractions. It should be noted that there is still antisymmetry under permutations of the $\{t\}$ or the $\{z\}$ separately, as there should be. It is quite surprising that a Wick theorem result holds in this case also.

2. The case $T<T_{C}, m=n=2$ was used in the theory of the interface between phases for the rectangular Ising ferromagnet [6].

\section{Representation of the $\boldsymbol{n}$-Point Function}

The following formula was developed in Paper I [1] of this series. The notation $(\boldsymbol{r})_{n}$ $=\left(\boldsymbol{r}_{1}, \ldots, \boldsymbol{r}_{n}\right)$ will be used for the location of the $n$ particles, with $\boldsymbol{r}_{j} \in \mathbb{Z}^{2}$. The relative coordinates are $x_{k}=\left(\boldsymbol{r}_{k+1}-\boldsymbol{r}_{k}\right) \cdot \boldsymbol{i}$ and $y_{k}=\left(\boldsymbol{r}_{k+1}-\boldsymbol{r}_{k}\right) \cdot \boldsymbol{j}$ where $\boldsymbol{i}$ and $\boldsymbol{j}$ are unit vectors for the lattice $\mathbb{Z}^{2}$ and $i$ is the transfer direction. The points are ordered so that $x_{k} \geqq 0, k=1, \ldots, n-1$. The $n$-point function is

$$
\begin{aligned}
\left\langle\sigma(\boldsymbol{r})_{n}\right\rangle= & \lim _{M \rightarrow \infty} \sum_{j_{1} \ldots j_{n+1}} \exp -\sum_{k=1}^{n-1}\left(\gamma\left(j_{k}\right) x_{k}-i \omega\left(j_{k}\right) y_{k}\right) \\
& \cdot\left\langle\Phi_{+}\left|\sigma_{1}^{x}\right| \Phi_{j_{1}}\right\rangle \prod_{1}^{n-2}\left\langle\Phi_{j_{l}}\left|\sigma_{1}^{x}\right| \Phi_{j_{l+1}}\right\rangle \\
& \cdot\left\langle\Phi_{j_{n-1}}\left|\sigma_{1}^{x}\right| \Phi_{+}\right\rangle .
\end{aligned}
$$

The index $j$ of each state $\left|\Phi_{j}\right\rangle$ is given by a set of wavenumbers $(\omega)_{m_{j}}$ with $m_{j} \geqq 0\left(m_{j}=0\right.$ corresponds to $\left.\left|\Phi_{+}\right\rangle\right)$with $\omega \in[0,2 \pi]$. The summations become integrations in the thermodynamic limit, as can be seen by considering Section 5 of 
[1], giving the result

$$
\begin{aligned}
\left\langle\sigma\left((\boldsymbol{r})_{n}\right)\right\rangle= & \sum_{m_{1}, \ldots, m_{n-1}=0}^{\infty} \int_{0}^{2 \pi} \ldots \int d\left(\omega_{1}\right)_{m_{1}} \ldots d\left(\omega_{n-1}\right)_{m_{n-1}} \prod_{1}^{n-1} \frac{1}{(2 \pi)^{m_{j}} m_{j} !} \\
& \cdot F_{x}\left(\left(e^{i \omega_{1}}\right)_{m_{1}}\right) \prod_{1}^{n-2} F_{x}\left(\left(e^{i \omega_{j}}\right)_{m_{j}} \mid\left(e^{i \omega_{j+1}}\right)_{m_{j+1}}\right) F_{x}\left(\left(e^{i \omega_{n-1}}\right)_{m_{n-1}}\right) \\
& \cdot \exp \sum_{l=1}^{n-1} \sum_{k=1}^{m_{l}}\left(-\gamma\left(\omega_{k l}\right) x_{l}+i y_{l} \omega_{k l} \operatorname{sgn} l\right),
\end{aligned}
$$

where the notation

$$
\left(\omega_{j}\right)_{n}=\left(\omega_{1 j}, \ldots, \omega_{n j}\right)
$$

will be used.

Just as for the 2-point function, there is an illuminating graphical representation of these results. For an $n$-point function consider vertex sets $\mathscr{R}_{j}$, $j=1, \ldots, n-1$. The $k^{\text {th }}$-vertex within $\mathscr{R}_{j}$ is labelled $\omega_{k j}, k=1, \ldots, m_{j}$ with $\left|\mathscr{R}_{j}\right|=m_{j}$, $\mathscr{R}_{j}$ is the set of wavenumbers describing $\left|\Phi_{j}\right\rangle$. For pictorial purposes it is convenient to arrange each $\mathscr{R}_{j}$ horizontally and then order the $\mathscr{R}_{j}$ vertically.

The union of the $\mathscr{R}_{j}$ will now be taken as the vertex set $V$ for a graph $\mathscr{G}=\{V, E\} ;$ the contractions $f_{ \pm}$which occur in Theorems 1 and 2 of the previous section will be assigned as edge weights on $\mathscr{G}$. Evidently there will be $f_{-}$edges within rows $\mathscr{R}_{j}$ but $f_{+}$edges between $\mathscr{R}_{j}$ and $\mathscr{R}_{j+1}$ for $j=1, \ldots, n-1$. First we rationalise the contraction functions so that the edge weights become real. By analogy with [2] we introduce the functions

$$
\begin{aligned}
& e_{ \pm}^{>}\left(\omega_{1}, \omega_{2}\right)=\left(\sinh \gamma\left(\omega_{1}\right) \pm \sinh \gamma\left(\omega_{2}\right)\right) / 2 \sin \left(\left(\omega_{1}+\omega_{2}\right) / 2\right), \\
& e_{ \pm}^{<}\left(\omega_{1}, \omega_{2}\right)=\left(p\left(\omega_{1}\right) q\left(\omega_{2}\right) \pm p\left(\omega_{2}\right) q\left(\omega_{1}\right)\right) / 2 \sin \left(\left(\omega_{1}+\omega_{2}\right) / 2\right) .
\end{aligned}
$$

with

$$
\begin{aligned}
& p(\omega)=(-2 \cos \omega+A+1 / A)^{1 / 2} \\
& q(\omega)=(-2 \cos \omega+B+1 / B)^{1 / 2}
\end{aligned}
$$

for the rectangular Ising model. The integration weight for each vertex is now

$$
d \mu(\omega)=d \omega / 2 \pi \sinh \gamma(\omega)
$$

and the factors of $i$ arising from the replacement of $f$ by $e$ can readily be shown to cancel.

Reference to Theorems 1 and 2 shows that the graphs in the two cases will be different. The case $T<T_{C}$ is the simpler: our considerations here will apply only to periodic boundary conditions, for which $\left\langle\sigma(\boldsymbol{r})_{n}\right\rangle=0$ whenever $n$ is odd (This is obviously not so with + boundary conditions: take $n=1)$. Allowed graphs $\mathscr{G}$ are unions of disjoint closed cycles $\mathscr{C}_{l}$. Each $\mathscr{C}_{l}$ has an even number of edges, weighted by $e_{-}^{<}(\cdot, \cdot)$ if both vertex labels come from the same $\mathscr{R}_{j}$. Within the vertical ordering $e_{+}^{<}(\cdot, \cdot)$ can only connect elements of $\mathscr{R}_{j}$ and $\mathscr{R}_{k}$ if $j=k \pm 1$. Closure of any $\mathscr{C}_{l}$ requires that the number of $e_{+}$weighted edges be even. The final problem here concerns the sign factors in the expansions over permutations. This is given by 
Lemma 1. Any closed cycle has a permutation factor of $(-1)$.

Proof. This is analogous to that in [2]. The only difference is that a product of $2 n-1$ permutations has to be handled because a cycle is permitted to intersect all $\mathscr{R}_{j}, j=1, \ldots, 2 n-1$.

When $T>T_{C}$, open chains occur. $\mathscr{R}_{1}$ and $\mathscr{R}_{2 n-1}$ have each one chain end arising from the first and last matrix elements respectively in (3.2). There is one in each $\mathscr{R}_{j} \cup \mathscr{R}_{j+1}$ for $j=1, \ldots, 2 n-2$ arising from the intermediate matrix elements. Each chain end has an edge emanating from it; the degree of all remaining vertices is two. Thus any allowed graph is a disjoint union of $n$ chains and any number of closed cycles. These are weighted in accordance with the rules for $T>T_{C}$, mutatis mutandis.

The permutation sign of a given chain is given by the following lemma:

Lemma 2. An open chain which has ends in $\mathscr{R}_{j}$ and $\mathscr{R}_{k}$ has an even (resp. odd) number of edges if $(j-k)$ is even (resp. odd). The permutation sign is $(-1)^{(j-k)}$.

Proof. This is an elementary extension of that in [2].

The final information required to specify the graphical representation is the vertex weight function for a vertex label $\omega_{j k}$ in row $\mathscr{R}_{k}$. This weight, denoted $v_{k}\left(\omega_{j k}\right)$ is given by

$$
v_{k}\left(\omega_{j k}\right)=\exp \left(-\left|x_{k}\right| \gamma\left(\omega_{j k}\right)+i y_{k} \omega_{j k} \operatorname{Sgn} k\right) .
$$

The appearance of $\operatorname{Sgn} k$ in (3.7) is a consequence of the choice of wavenumbers in (2.1) and (2.22). All spins are translated in the direction perpendicular to transfer to the standard position 1 in accordance with the procedures of [1].

The sums over appropriate weighted graphs for $T>T_{C}$ and $T<T_{C}$ are denoted $\varrho^{ \pm}\left(\left(x_{n-1},(y)_{n-1}\right)\right.$. The vacuum scalar products required as boundary conditions for the Pfaffian expansion of Theorems 1 and 2 are given in [2]. One obtains:

$$
\begin{aligned}
& T>T_{C}: \\
& \left\langle\sigma(\boldsymbol{r})_{2 n}\right\rangle=\left(\hat{m}\left(K_{1}, K_{2}\right) / \cosh K_{1}^{*}\right)^{2 n} \varrho^{+}\left((x)_{2 n-1},(y)_{2 n-1}\right),
\end{aligned}
$$

where

$$
\hat{m}\left(K_{1}, K_{2}\right)=\left(1-\left(\sinh 2 K_{1} \sinh 2 K_{2}\right)^{2}\right)^{1 / 8}
$$

and

$$
\begin{aligned}
& e^{-2 K_{1}^{*}}=\tanh K_{1} . \\
& T<T_{C}: \\
& \left\langle\sigma(\boldsymbol{r})_{2 n}\right\rangle=\left(m^{*}\left(K_{1}, K_{2}\right)\right)^{2 n} Q^{-}\left((x)_{2 n-1},(y)_{2 n-1}\right),
\end{aligned}
$$

where $m^{*}\left(K_{1}, K_{2}\right)$ is the spontaneous magnetisation, given first by Onsager [7]:

$$
m^{*}\left(K_{1}, K_{2}\right)=\left(1-\left(\sinh 2 K_{1}, \sinh K_{2}\right)^{-2}\right)^{1 / 8} \text {. }
$$

Remarks. 1. A conjecture has been given on the scaling limit of the truncated $n$ point functions [5] which suggests that the equation of state of the ising 
ferromagnet has an asymptotic form

$$
m(h, t) \sim t^{1 / 8} f\left(h t^{-15 / 8}\right)
$$

where $t=\left(T-T_{C}\right) / T_{C}$ and $h$ is the applied field. But the precise meaning of the symbol $\sim$ is yet to be given, as well as the properties and form of $f$.

2. Duneau et al. [3] have stressed the relationship between spanning tree decay properties of truncated $n$-point functions and analyticity. It appears difficult to establish such results rigorously. Study 4-point functions indicates that the occurrence of the contraction $f_{+}(\cdot, \cdot)$ is involved in an essential way in the truncation.

3. The results for $T<T_{C}$ have been obtained with toroidal boundary conditions. Messager and Miracle-Sole [8] have shown that below the critical temperature there are just two extremal invariant states $\omega_{ \pm}$; the state considered here is just $\left(\omega_{+}+\omega_{-}\right) / 2$. The results for $\omega_{+}$, and hence for any invariant equilibrium state will be given in another paper, using some of the methods of the last-named article in [7].

Acknowledgements. The author thanks the Aspen Center for Physics, the I.H.E.S., the Laboratoire de Physique Théorique of the EPF, Lausanne, and the Rockefeller University for hospitality and support while parts of this work were carried out. He is most grateful to Professors P. L. Choquard, M. L. Glasser, J. Glimm, J. Groeneveld, M. Kac, H. Kunz, and T. Spencer for discussions.

\section{References}

1. Abraham,D. B.: Commun. math. Phys. 59, 17 (1978)

2. Abraham,D.B.: Commun. math. Phys. 60, 181 (1978)

3. Duneau, M., Iagolnitzer, D., Souillard, B. : Commun. math. Phys. 31, 191 (1973)

4. Abraham,D.B.: Phys. Lett. 39 A, 375 (1972)

5. Abraham,D.B.: Phys. Lett. 61 A, 271 (1977)

6. Abraham,D.B., Reed,P.: Phys. Rev. Letters 33, 377 (1974); Commun. math. Phys. 49, 35 (1976); J. Phys. 10 A, 121 (1977)

7. Onsager,L.: Unpublished (1949). See also Yang,C.N.: Phys. Rev. 85, 808 (1952); Bennettin,G., Gallavotti,G., Jona-Lasinio, G., Stella, A.L. : Commun. math. Phys. 30, 45 (1973); Abraham,D.B., Martin-Löf, A. : Commun. math. Phys. 31, 245 (1973)

8. Messager,A., Miracle-Sole,S.: Commun. math. Phys. 40, 187 (1975)

Communicated by E. Lieb

Received February 20, 1978

\section{Note Added in Proof}

R. Z. Bariev [Phys. Lett. 64A, 169 (1977)] has derived $n$-point functions independently. 
